\title{
Oleic Acid and Hydroxytyrosol Inhibit Cholesterol and Fatty Acid Synthesis in C6 Glioma Cells
}

\author{
Paola Priore, ${ }^{1,2}$ Antonio Gnoni, ${ }^{3}$ Francesco Natali, ${ }^{1}$ Mariangela Testini, ${ }^{1}$ Gabriele V. Gnoni, ${ }^{1}$ \\ Luisa Siculella, ${ }^{1}$ and Fabrizio Damiano ${ }^{1}$ \\ ${ }^{1}$ Laboratory of Biochemistry and Molecular Biology, Department of Biological and Environmental Sciences and Technologies, \\ University of Salento, Via Prov.le Lecce-Monteroni, 73100 Lecce, Italy \\ ${ }^{2}$ CNR-NANOTEC, Institute of Nanotechnology clo Campus Ecotekne, University of Salento, Via Monteroni, 73100 Lecce, Italy \\ ${ }^{3}$ Department of Basic Medical Sciences, Neurosciences and Sense Organs, University of Bari "Aldo Moro", \\ Policlinico P.zza G. Cesare 11, 70100 Bari, Italy \\ Correspondence should be addressed to Luisa Siculella; luisa.siculella@unisalento.it
}

Received 26 May 2017; Revised 8 September 2017; Accepted 5 December 2017; Published 24 December 2017

Academic Editor: Tommaso Cassano

Copyright ( 2017 Paola Priore et al. This is an open access article distributed under the Creative Commons Attribution License, which permits unrestricted use, distribution, and reproduction in any medium, provided the original work is properly cited.

Recently, the discovery of natural compounds capable of modulating nervous system function has revealed new perspectives for a healthier brain. Here, we investigated the effects of oleic acid (OA) and hydroxytyrosol (HTyr), two important extra virgin olive oil compounds, on lipid synthesis in C6 glioma cells. OA and HTyr inhibited both de novo fatty acid and cholesterol syntheses without affecting cell viability. The inhibitory effect of the individual compounds was more pronounced if OA and HTyr were administered in combination. A reduction of polar lipid biosynthesis was also detected, while triglyceride synthesis was marginally affected. To clarify the lipid-lowering mechanism of these compounds, their effects on the activity of key enzymes of fatty acid biosynthesis (acetyl-CoA carboxylase-ACC and fatty acid synthase-FAS) and cholesterologenesis (3-hydroxy-3-methylglutaryl-CoA reductase-HMGCR) were investigated in situ by using digitonin-permeabilized C6 cells. ACC and HMGCR activities were especially reduced after $4 \mathrm{~h}$ of $25 \mu \mathrm{M}$ OA and HTyr treatment. No change in FAS activity was observed. Inhibition of ACC and HMGCR activities is corroborated by the decrease of their mRNA abundance and protein level. Our results indicate a direct and rapid downregulatory effect of the two olive oil compounds on lipid synthesis in C6 cells.

\section{Introduction}

Extra virgin olive oil (EVOO), the principal source of fat in the Mediterranean diet, represents the topic of many studies because several epidemiological data suggest that it positively affects human health, reducing the incidence of cancer, hypertension, and cardiovascular diseases $[1,2]$. Besides these wellrecognized effects, recent clinical studies support the efficacy of the Mediterranean diet and of its main fat also against the cognitive decline associated with ageing as well as against the onset and progression of a number of neurodegenerative diseases. In such neurological contexts, several data highlight the role of the natural compounds whose EVOO is rich $[3,4]$.
The EVOO health effects can be ascribed to a plethora of molecules contained in both its saponifiable (fatty acids) and unsaponifiable (mainly cholesterol) fractions. Initially, the beneficial properties of EVOO were attributed to its high content in oleic acid (OA). OA consumption was claimed to promote cardiovascular disease prevention and to influence the expression of homeostatic and metabolic genes that protect tissues from oxidative and inflammatory processes associated with ageing, degenerative diseases, and cancer [5-9]. In a previous study carried out on rat C6 glioma cells [5], it has been shown that, among a number of various fatty acids, OA was the most effective downregulator of lipid synthesis. 
In the last decade, a flurry of scientific findings has highlighted that many of the EVOO beneficial effects can be accounted not only for the monounsaturated nature of its predominant fatty acid OA but also for the bioactivity of EVOO minor compounds, which can act on cells through both indirect and direct mechanisms, the latter modulating gene expression [6, 8-10]. Among the minor constituents of EVOO, the phenolic compound hydroxytyrosol (2,(3,4-dihydroxyphenyl)-ethanol, HTyr) is considered one of the most effective antioxidants. Consumption of HTyr has certain health benefits, and the responsible mechanisms for these effects have been mainly attributed to its ability to scavenge reactive oxygen species and to enhance endogenous antioxidant systems [11]. In previous studies carried out on rat primary hepatocytes $[12,13]$, we found that HTyr (alone or as a part of a crude extract) stands out for its capacity to modulate also some enzyme activities relevant for lipid metabolism.

After white adipose tissue, the brain is the organ with the highest lipid content of the body. The biosynthesis and deposition of lipids play a pivotal role in maintaining the brain structure and function. Alterations in lipid metabolism are the cause of or are associated with many neurological diseases $[14,15]$.

Attenuation of age- and disease-associated cognitive decline by olive oil or by its minor compounds has been observed in cellular, animal, and human models [16-18]. In most of these studies, a reduction of oxidative damage and a modulation of antioxidant defences were shown. However, besides the antioxidant and anti-inflammatory activity, other mechanisms might underlie the beneficial effects of EVOO minor compounds on brain development and homeostasis [3, 16-18].

Considering the need for chemotherapeutic intervention against neurological disorders [19] and the putative role of fatty acids and antioxidants in this field [7, 15, 20], further studies are required to gain insight into the impact of EVOO compounds on neurodegenerative processes. C6 glioma cells present a large repertoire of astrocyte-expressing enzymatic activities $[21,22]$ and exhibit a prevalent astrocyte-like phenotype when cultured in serum-rich medium [23]. Thus, they are considered a useful cellular model to study cerebral dysfunction $[20,24]$.

To our knowledge, this is the first study in which the effect of EVOO constituents on lipid metabolism in glial cells has been investigated. In detail, this study was focused on the effects of the cosupplementation of OA and HTyr on cholesterol and fatty acid syntheses in glial cells.

\section{Materials and Methods}

2.1. Materials. Rat C6 glioma cells were from the American Type Culture Collection. Dulbecco's modified eagle's high-glucose medium (DMEM), foetal bovine serum (FBS), penicillin/streptomycin, phosphate buffer solution (PBS), and 3-[4,5-dimethylthiazol-2-yl]-2,5-diphenyltetrazolium bromide (MTT) were obtained from Gibco-Invitrogen Ltd. (Paisley, UK); $\left[1-{ }^{14} \mathrm{C}\right]$ acetate was obtained from GE Healthcare (Little Chalfont, UK); $\left[1-{ }^{14} \mathrm{C}\right]$ acetyl-CoA, $\left[3-{ }^{14} \mathrm{C}\right] 3$-hydroxy-3-methylglutaryl-CoA $\left(\left[3-{ }^{14} \mathrm{C}\right] \mathrm{HMG}-\mathrm{CoA}\right)$ were obtained from PerkinElmer (Boston, MA). Primary antibodies for acetyl-CoA carboxylase (ACC), fatty acid synthase (FAS), and $\alpha$-tubulin were obtained from Cell Signaling Technologies (Boston, MA). The antibody against 3-hydroxy-3-methylglutaryl-CoA reductase (HMGCR) as well as horseradish peroxidase-conjugated IgGs was obtained from Santa Cruz Biotechnology (Dallas, TX). All other reagents, obtained from Sigma-Aldrich, were of analytical grade.

2.2. Cell Culture. C6 cells were maintained in DMEM supplemented with $10 \%$ FBS and $1 \%$ penicillin/streptomycin, at $37^{\circ} \mathrm{C}$ in a humidified atmosphere of $5 \% \mathrm{CO}_{2}$. Unless otherwise specified in the text, $\mathrm{C} 6$ cells were seeded in 6-well plates (Corning Inc., Corning, NY) at a density of $5 \times 10^{5}$ cells per well and cultured in 10\% FBS-supplemented medium. $24 \mathrm{~h}$ after plating, the medium was changed and, following further $24 \mathrm{~h}$, OA sodium salt and HTyr were added for $4 \mathrm{~h}$, singularly or in coincubation, to the DMEM medium, obtaining $25 \mu \mathrm{M}$ final concentration. OA stock solution was $10 \mathrm{mM}$ in DMEM and HTyr stock solution was $100 \mathrm{mM}$ dissolved in dimethyl sulfoxide (DMSO). For each determination, untreated control cells were also considered.

2.3. Cell Viability Assay. Cell proliferation and viability were assessed by the MTT assay. To this purpose, C6 cells were cultured at a density of $5 \times 10^{3}$ cells/well in a 96-well plate (Corning Inc., Corning, NY) and after $24 \mathrm{~h}$, the serum-rich medium was refreshed. Following further $24 \mathrm{~h}$, cells were incubated for $4 \mathrm{~h}$ with OA and/or HTyr, using for each sample three concentrations: $25 \mu \mathrm{M}, 50 \mu \mathrm{M}$, and $100 \mu \mathrm{M}$ of $\mathrm{OA}$ and/or the HTyr. Then, cell monolayers were incubated for $3 \mathrm{~h}$ with $1 \mathrm{mg} / \mathrm{mL}$ MTT. Mitochondria of living cells transform the yellow-coloured tetrazolium compound to its purple formazan derivative. Formazan crystals formed in the cells were dissolved in $100 \mu \mathrm{L}$ DMSO, and the absorbance was measured at $570 \mathrm{~nm}$ using a Multiskan FC ELISA reader (Thermo Fisher Scientific, Waltham, MA). The viability is calculated as percentage of absorbance relative to control cells.

2.4. Rate of Fatty Acid and Cholesterol Synthesis. Acetyl-CoA is the precursor for both fatty acid and cholesterol synthesis. Lipogenic activity was monitored by the incorporation of $\left[1-{ }^{14} \mathrm{C}\right]$ acetate $(16 \mathrm{mM}, 0.96 \mathrm{mCi} / \mathrm{mol})$ into total fatty acids and cholesterol essentially as in Gnoni et al. [25]. Cells were incubated for $4 \mathrm{~h}$ with $25 \mu \mathrm{M}$ OA and/or $25 \mu \mathrm{M}$ HTyr. Labelled acetate was added $1 \mathrm{~h}$ before ending the experiment.

To terminate the lipogenic assay, the medium was aspirated and the cells were washed three times with ice-cold PBS to remove unreacted acetate, and the reaction was stopped by $1.5 \mathrm{~mL}$ of $0.5 \mathrm{~N} \mathrm{NaOH}$.

The cells were scraped off, transferred to a test tube, and saponified with ethanolic $\mathrm{KOH}$. Sterols and fatty acids were extracted and counted for radioactivity as reported [25].

2.5. Chromatographic Analysis of Radiolabelled Lipid Fractions. Radiolabelled acetate incorporation into phospholipids and neutral lipids was analyzed. At the end of the 
incubation period, the cells were washed with ice-cold PBS and the reaction was blocked with $2 \mathrm{~mL}$ of $\mathrm{KCl}: \mathrm{CH}_{3} \mathrm{OH}$ $(1: 2, v / v)$. Total lipids were extracted according to Bligh and Dyer [26] and resolved by thin layer chromatography on silica gel plates, using as developing system $\mathrm{CHCl}_{3}: \mathrm{CH}_{3} \mathrm{OH}: 28 \% \mathrm{NH}_{4} \mathrm{OH}(65: 25: 4)$ and exane: ethyl ether:acetic acid $(80: 20: 1)$ for phospholipids and neutral lipid analysis, respectively. Lipid spots were visualized with iodine vapour and scraped into counting vials for radioactivity measurement [5].

\subsection{Chromatographic Analysis of Radiolabelled Fatty Acids.} HPLC analysis of the extracted fatty acids was performed as reported [5]. $20 \mu \mathrm{L}$ of sample was injected into a Beckman Coulter System Gold Programmable Solvent Module 125 and furnished with a C18 ODS column $(4.6 \times 250 \mathrm{~mm})$ and Diode Array Detector 168 (Beckman Coulter, Milan, IT). Two mobile phases were used for elution: solvent A, constituted by acetonitrile: water $(4: 1)$ and ran for $45 \mathrm{~min}$ and solvent $B$, constituted by acetonitrile and ran for another $15 \mathrm{~min}$. Flow rate was $2 \mathrm{~mL} / \mathrm{min}$ and detection was at $242 \mathrm{~nm}$. Eluted fractions were collected for radioactivity measurement.

2.7. Assay of Lipogenic Enzyme Activities. ACC activity was determined as the incorporation of radiolabelled acetylCoA into fatty acid in an assay coupled with FAS activity. This method circumvents interferences linked to the classical bicarbonate assay [27]. ACC and FAS activities were determined in digitonin-permeabilized C6 cells. Cell permeabilization, achieved by using an assay mix containing $400 \mu \mathrm{g} / \mathrm{mL}$ digitonin [5], represents an appropriate tool to investigate enzyme activities directly in situ (i.e., in a more or less natural environment).

FAS activity was assayed by measuring the incorporation of $\left[1-{ }^{14} \mathrm{C}\right]$ acetyl-CoA into fatty acids essentially as described above for ACC activity, except that $0.2 \mathrm{mM}$ malonyl-CoA was included and ATP, butyryl-CoA and FAS were omitted in the digitonin-containing assay mixture [28]. The assay was carried out at $37^{\circ} \mathrm{C}$ for $10 \mathrm{~min}$.

Both the lipogenic assays were stopped by the addition of $100 \mu \mathrm{L}$ of $10 \mathrm{M} \mathrm{NaOH}$. The samples were saponified by adding $5 \mathrm{~mL}$ of $\mathrm{CH}_{3} \mathrm{OH}$ and boiling for 45-60 min in capped tubes. After acidification with $200 \mu \mathrm{L}$ of $12 \mathrm{M} \mathrm{HCl}$, fatty acids were extracted and counted for the radioactivity as in [5].

The activities of ACC and FAS are expressed as nanomoles of $\left[1-{ }^{14} \mathrm{C}\right]$ acetyl-CoA incorporated into fatty acids per minute per milligram of protein.

\subsection{Activity Assay of 3-Hydroxy-3-Methylglutaryl-CoA} Reductase (HMGCR). HMGCR is the rate-controlling enzyme in the biosynthesis of cholesterol. The HMGCR activity assay was carried out essentially as described in [5]. Briefly, C6 cells were seeded at a density of $2 \times 10^{6}$ cells per $100 \mathrm{~mm}$ diameter Petri dish. After $48 \mathrm{~h}, 25 \mu \mathrm{M}$ OA and HTyr were added singularly or in coincubation to the medium for $4 \mathrm{~h}$. Then, the medium was discarded and the cells were scraped into a buffer containing $50 \mathrm{mM}$ Tris- $\mathrm{HCl}(\mathrm{pH} 7.4)$ and $150 \mathrm{mM} \mathrm{NaCl}$. After centrifugation $(900 \times \mathrm{g}, 3 \mathrm{~min}$, room temperature), the pellet was frozen in liquid nitrogen and kept at $-80^{\circ} \mathrm{C}$ until use.

Cell extracts were prepared from the pellets which were thawed, resuspended, and subsequently used for HMGCR activity assay [5]. The reaction started by the addition of $\left[3-{ }^{14} \mathrm{C}\right] \mathrm{HMG}-\mathrm{CoA}(75 \mu \mathrm{M}, 1.8 \mathrm{Ci} / \mathrm{mol})$. After $120 \mathrm{~min}$ at $37^{\circ} \mathrm{C}$, the reaction was stopped by the addition of $20 \mu \mathrm{L}$ of $7 \mathrm{M} \mathrm{HCl}$. Conversion to mevalonolactone occurred following an additional $60 \mathrm{~min}$ incubation at $37^{\circ} \mathrm{C}$ and the radioactive product was isolated by TLC, using toluene: acetone $(1: 1)$ as the mobile phase. Spots were collected and counted for the radioactivity. As internal standard, $\left[{ }^{3} \mathrm{H}\right]$ mevalonolactone was used.

2.9. Isolation of RNA from C6 Cells and Real-Time $q P C R$ Analysis. Total RNA from C6 cells was isolated using the SV Total RNA Isolation System kit (Promega), following the manufacturer's instructions. The reverse transcriptase reaction $(20 \mu \mathrm{l})$ was carried out using $5 \mu \mathrm{g}$ of total RNA, $100 \mathrm{ng}$ of random hexamers, and 200 units of SuperScript III RNase H-Reverse Transcriptase (Life Technologies-Thermo Fisher Scientific, Waltham, MA) [29].

Quantitative gene expression analysis was performed using SYBR ${ }^{\circledR}$ Select Master Mix for CFX (Life Technologies-Thermo Fisher Scientific, Waltham, MA) and 18S rRNA for normalization. The primers used for quantitative real-time PCR analysis were as follows $\left(5^{\prime}\right.$ to $\left.3^{\prime}\right)$ : rFASNfor CTCTGGTGGTGTCTACATTTC; rFASNrev GAGCTCTT TCTGCAGGATAG; rACCfor CTTGGAGCAGAGAACC TTCG; rACCrev; CCTGGATGGTTCTTTGTCCC; rHMG CRfor CTCACAGGATGAAGTAAGGG; rHMGCRrev CT GAGCTGCCAAATTGGACG [30]

2.10. Western Blotting Analysis. Cells grown in 6-well dishes were treated with OA and/or HTyr as indicated above and lysed as previously described [12]. The extracts were boiled for $5 \mathrm{~min}$ and samples containing an equal amount of total protein $(25 \mu \mathrm{g})$ were loaded on $10 \%$ SDS-polyacrylamide gels. Following electrophoresis, the proteins were transferred onto a nitrocellulose membrane [13]. To detect ACC, FAS, and HMGCR, membranes were incubated with the specific primary antibodies for $1.5 \mathrm{~h}$ at room temperature and then for $1 \mathrm{~h}$ with appropriate horseradish peroxidase-conjugated IgG (dilution 1:5000). Signals were detected by enhanced chemiluminescence using the Amersham ECL plus kit (GE Healthcare, Milan, Italy). Beta-actin detection was used for signal normalization.

\section{Statistical Analysis}

Data are the means \pm S.D. for the indicated number of experiments. The results were computed with Excel (Microsoft 10). Comparisons among groups were made using one-way analysis of variance (ANOVA). The differences between mean values were tested, using Bonferroni post hoc test. All statistical analyses were performed by GraphPad Prism 6 software (GraphPad Software Inc., La Jolla, CA). Differences were considered statistically significant at $P<0.05$. 


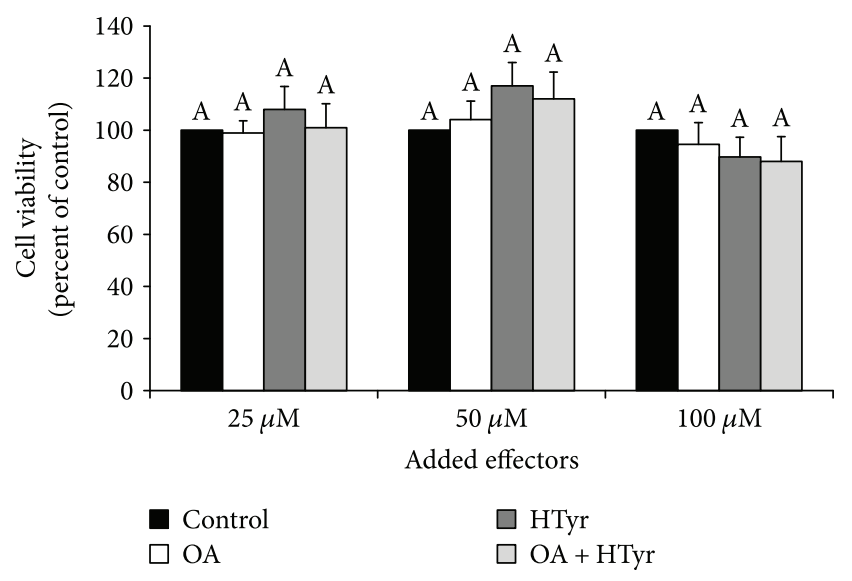

Figure 1: Effect of oleic acid and/or hydroxytyrosol on C6 cell viability. C6 cells were incubated for $4 \mathrm{~h}$ with $25 \mu \mathrm{M}, 50 \mu \mathrm{M}$, and $100 \mu \mathrm{M} \mathrm{OA}, \mathrm{HTyr}$, or their combination in serum-rich medium. Cell viability was estimated by an MTT assay. Values, expressed as $\%$ of control, are means \pm S.D. of five experiments. Within the same group, samples bearing different letters differ significantly $(P<0.05)$. Control: untreated cells; OA: oleic acid; HTyr: hydroxytyrosol.

\section{Results}

4.1. Cell Viability. MTT test showed that C6 cells incubated with OA or HTyr $(25 \mu \mathrm{M}, 50 \mu \mathrm{M}$, and $100 \mu \mathrm{M}$ for $4 \mathrm{~h})$, had the same viability of the control cells at each tested concentration (Figure 1). Also, the coincubation of OA and HTyr did not exert any cytotoxic effect. These findings were corroborated by morphological observation, protein assay, and trypan blue exclusion (data not shown). Thus, all further experiments were performed on cells treated with $25 \mu \mathrm{M}$ OA, $25 \mu \mathrm{M}$ HTyr, or their combination and incubated for $4 \mathrm{~h}$ in order to exclude putative nonspecific toxic effect while at the same time using the lowest effective concentration.

4.2. Effect of OA, HTyr, and Their Combination on Cholesterol and Fatty Acid Syntheses. Acetate in the cell is transformed into acetyl-CoA, which represents a common precursor for both fatty acid and cholesterol synthesis. Hence, both these metabolic pathways were simultaneously followed by using labelled acetate as a precursor.

Bar graphs in Figure 2 show a significant reduction of $\left[1-{ }^{14} \mathrm{C}\right]$ acetate incorporation into total cholesterol (Figure 2(a)) and fatty acids (Figure 2(b)). In particular, when C6 cells were incubated for $4 \mathrm{~h}$ with OA or HTyr, a decrease by $24 \%$ and $18 \%$, respectively, of $\left[1-{ }^{14} \mathrm{C}\right]$ acetate incorporation into cholesterol was observed. This inhibition was much more evident ( $-36 \%$ versus untreated cells) if OA and HTyr were added in combination to the cells.

With respect to cholesterologenesis, fatty acid synthesis was greater affected by EVOO compounds under investigation. Incubation of C6 cells singularly with OA, or HTyr led to a reduction of the radiolabelled acetate incorporation into fatty acids by about $56 \%$ and $23 \%$, respectively, compared to that measured in control cells. Analogously to cholesterol synthesis, fatty acid synthesis inhibition was more pronounced ( $-68 \%$ versus untreated cells) after $4 \mathrm{~h}$ of OA and HTyr coincubation of C6 cells.

\subsection{Effect of EVOO Components on Radiolabelled Acetate} Incorporation into Phospholipids and Neutral Lipids. Since newly synthesized fatty acids are mainly incorporated into complex lipids, the effect of OA and HTyr addition to C6 glioma cells on $\left[1-{ }^{14} \mathrm{C}\right]$ acetate incorporation into polar and neutral lipids was tested (Table 1). A general decrease of labelled precursor incorporation into all phospholipids, particularly into phosphatidylcholine, the most abundant phospholipid in C6 glioma cells, was observed mainly when cells were incubated for $4 \mathrm{~h}$ with OA and HTyr in combination. Among neutral lipids, unesterified fatty acids, cholesterol, and cholesterol ester were the fractions showing significant reduction in radioactivity incorporation due to the EVOO compound addition. Interestingly, only slight reduction in the incorporation of labelled acetate into triglycerides (TG) was detected after additions of OA and HTyr.

4.4. Analysis of Newly Synthesized Radiolabelled Fatty Acids. In order to investigate the effect of OA and HTyr additions to $\mathrm{C} 6$ cells on the individual fatty acids synthesized from labelled acetate, an HPLC analysis of the total fatty acid extract was carried out.

Figure 3 shows that, in agreement with previous results [5], in control cells, the incorporation of labelled acetate into the individual fatty acids was in the following order: palmitic acid (C16:0) > stearic acid (C18:0) > oleic acid (C18:1). Only a small amount of radioactivity was incorporated into other fatty acids (data not shown). A reduction of about $50 \%$ of the radiolabelled incorporation into palmitic, stearic, and oleic acid was observed upon OA addition to the cells, while a near 30\% decrease was evidenced upon HTyr treatment. The inhibitory effect of $\left[1-{ }^{14} \mathrm{C}\right]$ acetate incorporation into the fatty acids was more pronounced (about 70\%) when both $\mathrm{OH}$ and HTyr were contemporaneously added to the medium culture.

4.5. Modulation of Activity of ACC, FAS, and HMGCR by OA, HTyr, and Their Combination. Palmitic acid and to a lesser extent stearic acid are the main final products of the de novo fatty acid synthesis.

In order to determine the enzymatic steps of lipid biosynthetic pathways affected by the addition of OA, HTyr, and their combination, experiments were carried out to assay the activities of the key enzymes ACC and FAS for de novo fatty acid synthesis and HMGCR for cholesterol synthesis. All enzymatic activities were measured by in situ assays using digitonin-permeabilized C6 cells.

$4 \mathrm{~h}$ incubation of C6 cells with $25 \mu \mathrm{M}$ of OA or HTyr caused a reduction of ACC activity by about $45 \%$ and $19 \%$, respectively (Figure 4). A further decrement (-56\% versus untreated cells) was measured when these compounds were coincubated.

OA or HTyr lowered HMGCR activity by $29 \%$ and $16 \%$, respectively. During coincubation, the inhibition was more pronounced. In fact, HMGCR activity reached $61 \%$ of 


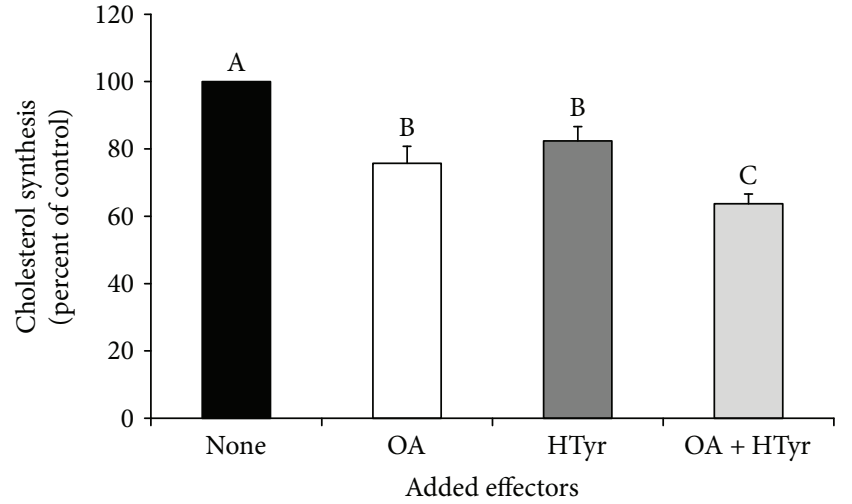

(a)

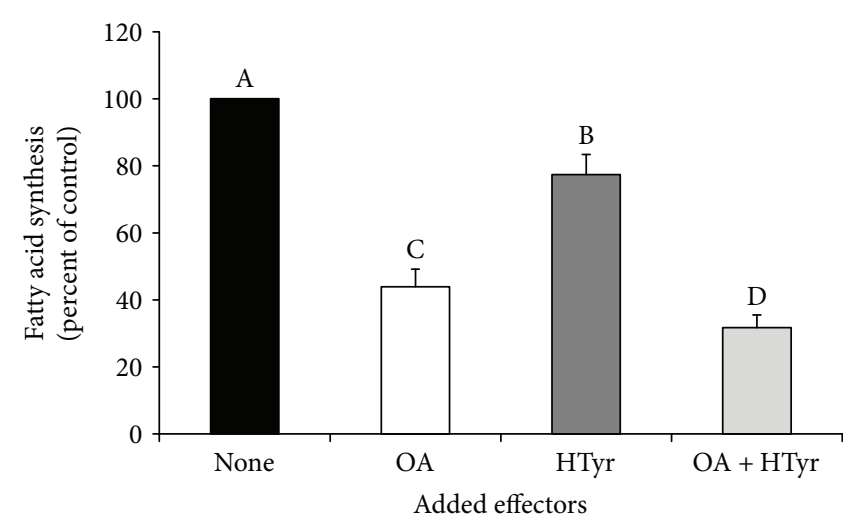

(b)

Figure 2: Modulation of cholesterol and fatty acid syntheses by oleic acid and/or hydroxytyrosol. After an initial $48 \mathrm{~h}$ plating, C6 glioma cells, growing in serum-rich medium, were incubated for $4 \mathrm{~h}$ with $25 \mu \mathrm{M}$ OA and/or $25 \mu \mathrm{M}$ HTyr. During the last hour of incubation, labelled acetate was added and its incorporation into cholesterol (a) and fatty acids (b) was followed. Data, nmol $\left[1-{ }^{14} \mathrm{C}\right]$ acetate/h/mg protein, are expressed as $\%$ of control and are means \pm S.D. of six independent experiments. In each experiment, determinations were carried out in triplicate. In control cells, rates of cholesterol and fatty acid synthesis were $1.43 \pm 0.07$ and $8.67 \pm 0.49 \mathrm{nmol}\left[1-{ }^{14} \mathrm{C}\right]$ acetate $\mathrm{inc} / \mathrm{h} / \mathrm{mg}$ protein, respectively. Samples bearing different letters differ significantly $(P<0.05)$. None: no addition to the cells; OA: oleic acid; HTyr: hydroxytyrosol.

TABLE 1: Effect of OA and HTyr and their combination on $\left[1-{ }^{14} \mathrm{C}\right]$ acetate incorporation into various lipid fractions in C6 cells

\begin{tabular}{|c|c|c|c|c|}
\hline Added effectors & None & $\mathrm{OA}$ & HTyr & $\mathrm{OA}+\mathrm{HTyr}$ \\
\hline \multicolumn{5}{|l|}{ Polar lipids } \\
\hline $\mathrm{CL}+\mathrm{PE}$ & $2151 \pm 151^{\mathrm{a}}$ & $1377 \pm 85^{\mathrm{b}}$ & $1742 \pm 121^{\mathrm{c}}$ & $1119 \pm 75^{\mathrm{d}}$ \\
\hline PC & $15,908 \pm 875^{\mathrm{a}}$ & $5596 \pm 308^{\mathrm{b}}$ & $12,781 \pm 703^{c}$ & $3487 \pm 192^{\mathrm{d}}$ \\
\hline SM & $741 \pm 67^{\mathrm{a}}$ & $463 \pm 37^{\mathrm{b}}$ & $640 \pm 37^{\mathrm{a}}$ & $270 \pm 19^{c}$ \\
\hline $\mathrm{PS}+\mathrm{PI}$ & $3781 \pm 246^{\mathrm{a}}$ & $1439 \pm 101^{\mathrm{b}}$ & $2533 \pm 165^{\mathrm{c}}$ & $962 \pm 65^{\mathrm{d}}$ \\
\hline \multicolumn{5}{|l|}{ Neutral lipids } \\
\hline MG & $289 \pm 20^{\mathrm{a}}$ & $231 \pm 11^{\mathrm{b}, \mathrm{c}}$ & $252 \pm 11^{\mathrm{b}}$ & $214 \pm 7^{\mathrm{c}}$ \\
\hline DG & $711 \pm 136^{\mathrm{a}}$ & $587 \pm 24^{\mathrm{a}}$ & $623 \pm 27^{\mathrm{a}}$ & $562 \pm 35^{\mathrm{a}}$ \\
\hline Cholesterol & $2155 \pm 194^{\mathrm{a}}$ & $1724 \pm 155^{\mathrm{b}}$ & $1896 \pm 170^{\mathrm{a}, \mathrm{b}}$ & $1509 \pm 136^{\mathrm{b}}$ \\
\hline Unesterified fatty acids & $239 \pm 14^{\mathrm{a}}$ & $200 \pm 12^{\mathrm{a}, \mathrm{b}}$ & $187 \pm 11^{\mathrm{b}}$ & $123 \pm 8^{\mathrm{b}}$ \\
\hline TG & $1123 \pm 73^{\mathrm{a}}$ & $1048 \pm 68^{\mathrm{a}, \mathrm{b}}$ & $999 \pm 65^{\mathrm{a}, \mathrm{b}}$ & $921 \pm 60^{\mathrm{b}}$ \\
\hline $\mathrm{CE}$ & $896 \pm 63^{\mathrm{a}}$ & $659 \pm 46^{\mathrm{b}, \mathrm{c}}$ & $725 \pm 47^{\mathrm{a}, \mathrm{b}}$ & $587 \pm 23^{\mathrm{c}}$ \\
\hline
\end{tabular}

C6 cells were incubated with $25 \mu \mathrm{M}$ oleic acid (OA) and/or $25 \mu \mathrm{M}$ hydroxytyrosol (HTyr) for $4 \mathrm{~h}$, and labeled acetate was added $1 \mathrm{~h}$ before ending the incubation. Total lipids were extracted. Phospholipids and neutral lipids were resolved by TLC, and the radioactivity associated with the different lipid fractions was counted. CL: cardiolipin; PE: phosphatidylethanolamine; PC: phosphatidylcholine; SM: sphingomyelin; PS: phosphatidylserine; PI: phosphatidylinositol; MG: monoglycerides; DG: diglycerides; TG: triglycerides; CE: cholesterol esters. Values are expressed as $\mathrm{cpm} / \mathrm{mg}$ protein $\pm \mathrm{SD}, n=5$. Within the same group, samples bearing different letters differ significantly $(P<0.05)$.

that observed in control cells in presence of $25 \mu \mathrm{M}$ OA and $25 \mu \mathrm{M}$ HTyr.

Notably, FAS activity was not significantly affected by either incubation conditions.

The reduced activity of ACC and of HMGCR is in accordance with the results of Figure 2, regarding the reduction of total synthesis of fatty acids (Figure 2(a)) and cholesterol (Figure 2(b)) starting from $\left[1-{ }^{14} \mathrm{C}\right]$ acetate.

4.6. Regulation of ACC, FAS, and HMGCR Expression by OA, HTyr and $\mathrm{OA}+H T y r$. Next, the molecular mechanisms responsible for the modulation exerted by OA or HTyr on the above-reported enzyme activities were investigated. To this aim, the abundance of mRNAs encoding for ACC,
FAS, and HMGCR was quantified by real-time qPCR analysis, and the amount of the corresponding encoded protein was determined by Western blotting.

In treated cells, a decrease of ACC mRNA abundance was observed (Figure 5(a)). After OA incubation, ACC mRNA level decreased by about $42 \%$ with respect to that measured in control cells; HTyr singularly added to the cell culture medium exerted minor inhibitory effect. In all the experimental conditions tested, no significant change in the abundance of FAS mRNA was detected (Figure 5(a)). After $4 \mathrm{~h}$ incubation with $25 \mu \mathrm{M}$ OA alone or in combination with $25 \mu \mathrm{M}$ HTyr, the amount of HMGCR mRNA lowered by about $18 \%$ and $22 \%$, respectively (Figure 5(a)). Results obtained for ACC, FAS, and HMGCR mRNA abundance 


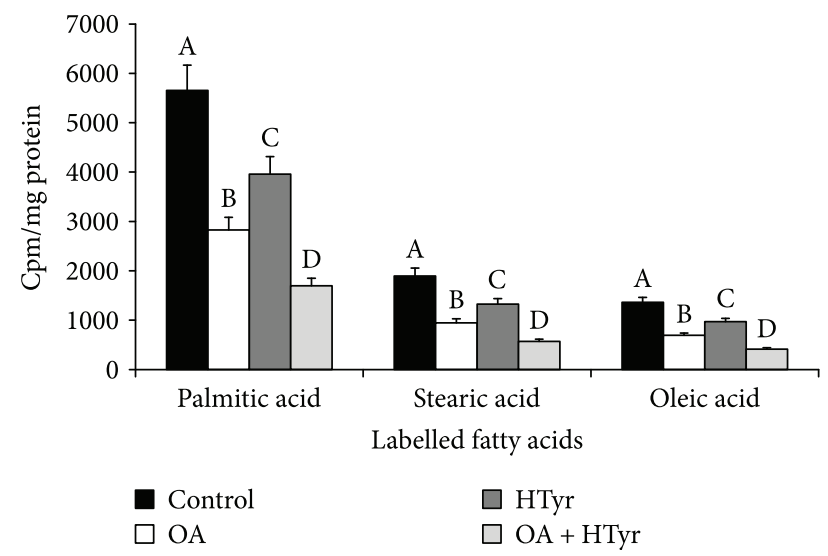

Figure 3: Effect of oleic acid, hydroxytyrosol, or their combination on $\left[1-{ }^{14} \mathrm{C}\right]$ acetate incorporation into individual fatty acids. The effects of $25 \mu \mathrm{M}$ OA, $25 \mu \mathrm{M}$ HTyr, and their combination on the incorporation of labelled acetate into different fatty acids were assayed. After $4 \mathrm{~h}$ of incubation, the radiolabelled neosynthesized fatty acids were extracted and separated by HPLC. Eluted fractions, corresponding to the different fatty acids, were collected for radioactivity measurement. Data, expressed as $\mathrm{cpm} / \mathrm{mg}$ protein, represent means \pm S.D. of six experiments. Within the same group, samples bearing different letters differ significantly $(P<0.05)$. OA: oleic acid; HTyr: hydroxytyrosol.

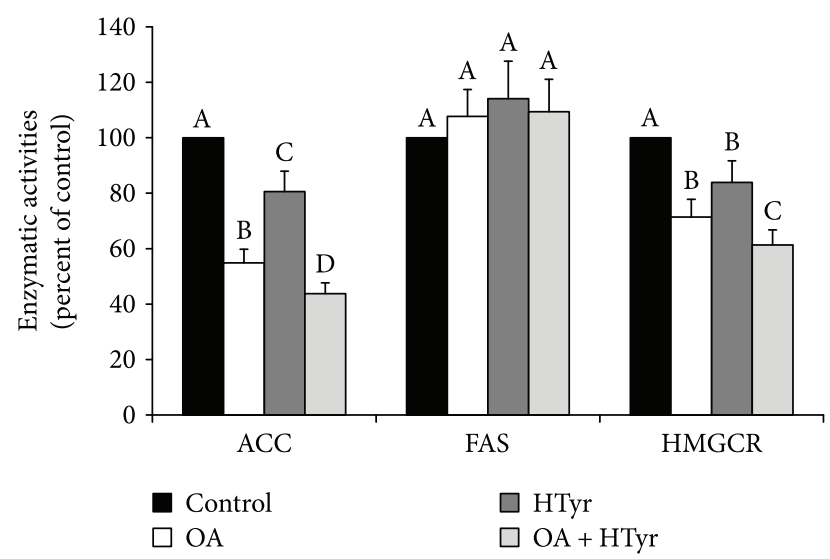

FIGURE 4: Oleic acid and/or hydroxytyrosol modulation of ACC, FAS, and HMGCR activities. After $4 \mathrm{~h}$ incubation with $25 \mu \mathrm{M}$ of $\mathrm{OA}$, HTyr, or OA + HTyr, the indicated enzyme activities were assayed in digitonin-permeabilized C6 cells. Values, expressed as percentage of control, are means \pm SD of five independent experiments. Control-specific activities were ACC, $0.178 \pm 0.011$ $\left[1-{ }^{14} \mathrm{C}\right]$ acetyl-CoA inc/min $/ \mathrm{mg}$ protein; FAS, $0.051 \pm 0.003 \mathrm{nmol}$ $\left[1-{ }^{14} \mathrm{C}\right]$ acetyl-CoA inc/min/mg protein; HMGCR, $33.6 \pm 1.9 \mathrm{pmol}$ $\left[3-{ }^{14} \mathrm{C}\right] \mathrm{HMG}-\mathrm{CoA}$ inc/min/mg protein. Within the same group, samples bearing different letters differ significantly $(P<0.05)$. OA: oleic acid; HTyr: hydroxytyrosol.

were confirmed also by the Western blotting analysis of the respective protein contents (Figure 5(b)).

\section{Discussion}

The Mediterranean diet has been considered the healthier dietary regimen, related to a reduced risk of several pathologies

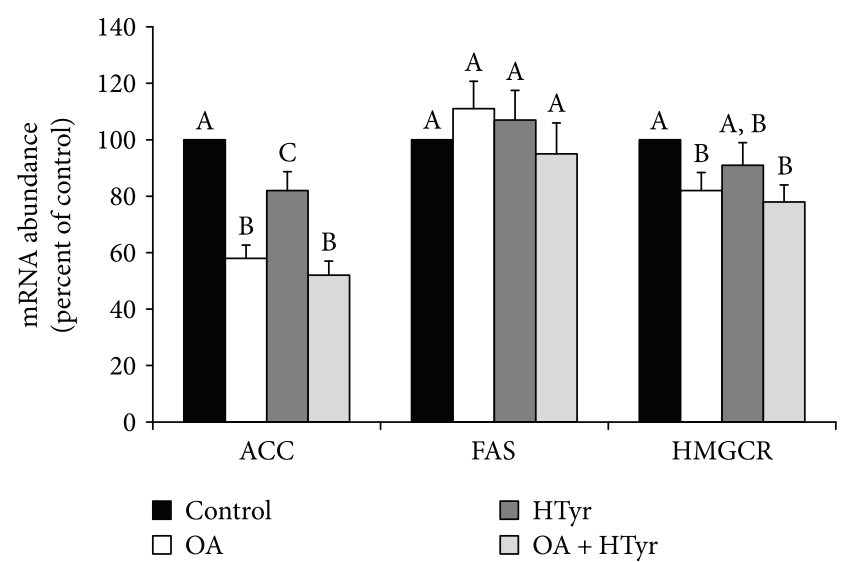

(a)
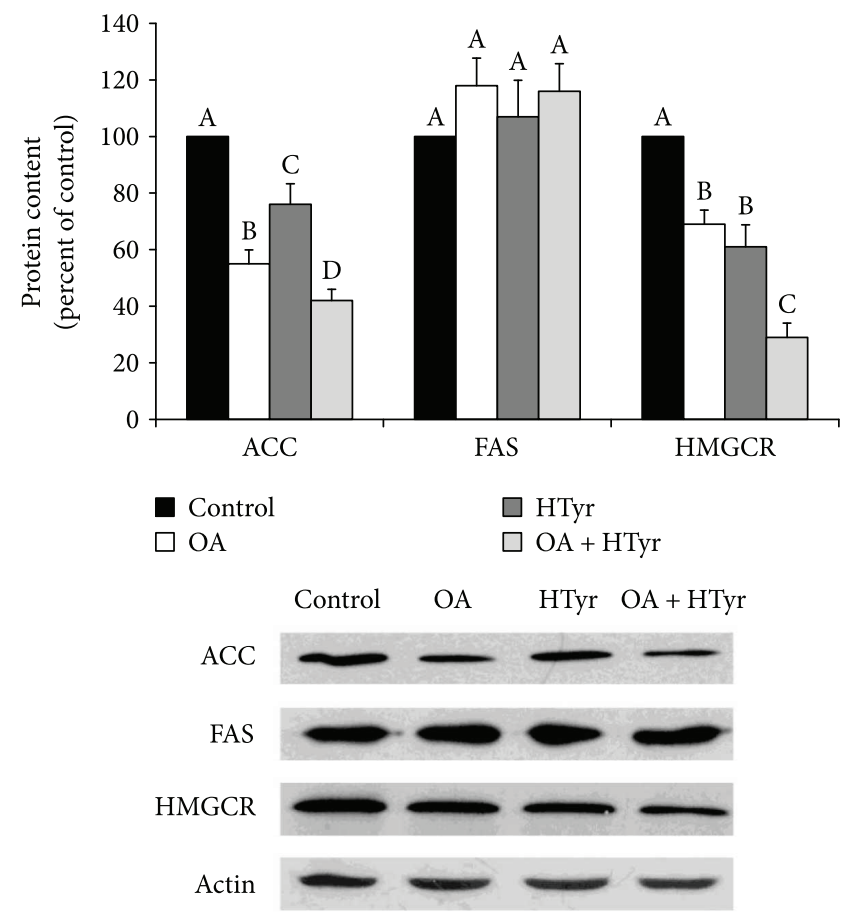

(b)

FIgURE 5: Effect of oleic acid, hydroxytyrosol, or their combination on ACC, FAS, and HMGCR mRNA abundance and protein content in C6 glioma cells. C6 cells were incubated with $25 \mu \mathrm{M}$ of OA, HTyr, or OA + HTyr for $4 \mathrm{~h}$ in serum-rich medium. (a) The abundance of ACC, FAS, and HMGCR mRNA was determined by RT-qPCR and normalized with respect to $18 \mathrm{~S}$ rRNA, used as a reference. Normalized values are expressed in histograms as percentage of the control. Values are means \pm S.D. of triplicate samples from each of four independent experiments. (b) C6 cells were lysed and protein content was isolated. ACC, FAS, and HMGCR were then assessed by Western blotting and quantified by densitometry. The protein contents are expressed as percentage of the control and are means \pm SD of three independent experiments. Within the same group, samples bearing different letters differ significantly $(P<0.05)$. OA: oleic acid; HTyr: hydroxytyrosol. 
such as metabolic disorders and cardiovascular diseases $[1,2$, $6,31,32]$. A key component of the Mediterranean diet is the EVOO, which is characterized by the presence of bioactive phytonutrients, with antioxidant and anti-inflammatory properties, and of monounsaturated fatty acid (OA) as fat source.

Studies from a number of research groups have established that variations in dietary fatty acids or in the intake of phenolic compounds are able to influence cellular metabolism and regulatory processes in neuronal and glial cells $[3,5,17,24]$, supporting mounting evidence that EVOO active components could have a great potential to reduce the incidence of neurodegenerative diseases $[3,18,33]$. It has to be stressed that most of these studies focused mainly on the antioxidant and anti-inflammatory activities of fatty acids [7] and of phenolic compounds [20], without taking into account their possible direct action on metabolism, such as lipid biosynthesis.

Lipids are fundamental components of neuronal membranes and are essential for brain function. Cholesterol and fatty acids are particularly present in the synaptic membranes and play a key role in the membrane fluidity and in the formation of specialized microdomains, lipid rafts, essential for synaptic transmission [34].

Brain lipids can derive from blood and/or can be endogenously synthesized. Fatty acids can cross the blood-brain barrier by a complex process, involving diffusional and protein-mediated transport, which occurs predominantly by fatty acid transport protein-1 and protein-4 (FATP-1 and FATP-4) in humans and mouse [34]. Lipid synthesis is inefficient in neurons but not in glia cells. In vitro studies have demonstrated that astrocytes, the most abundant glia cells, synthesize and release lipids that are complexed to apolipoprotein E- (ApoE-) containing lipoproteins [35]. Consequently, neuronal functions are negatively affected by perturbed lipid metabolism which has been observed in several neurological disorders, such as Niemann-Pick's disease [36], Alzheimer's disease [37], Huntington's disease [38], Parkinson's disease [39], and amyotrophic lateral sclerosis [40].

Despite the crucial role of EVOO bioactive compounds in brain function and metabolism, little is known about their action on lipogenesis in glial cells [5].

This work primarily shows that both fatty acid and cholesterol syntheses are rather active in cultured glioma C6 cells when $\left[1-{ }^{14} \mathrm{C}\right]$ acetate is used as common precursor for both the metabolic pathways, thus adding further support to previous findings [5]. Notably, in human malignant glial cells, compared with their normal counterparts, a very active de novo fatty acids and cholesterol syntheses have been reported [41].

The present study represents the first report of a direct and rapid effect of EVOO main compounds (OA and HTyr) on lipid synthesis in rat glioma cells. We show that in C6 cells, the addition of OA and HTyr causes a fast (within $4 \mathrm{~h}$ ) inhibition of radiolabelled acetate incorporation into both cholesterol and fatty acid fractions. A rapid effect of EVOO components on both these pathways has been described in different cell cultures [12, 13]. Beside the OA, a monounsaturated fatty acid, which represents the most abundant fatty acid (about 70\%) in EVOO, polyunsaturated fatty acids (such as linoleic acid), and saturated fatty acid (mainly palmitic acid) are significantly present in the fatty acid fraction of EVOO. However, Natali et al. reported that OA was the most effective in reducing lipid synthesis in C6 cells [5].

Compared to control cells, the radiolabelled precursor incorporation into cholesterol decreased by about $30 \%$ in cells incubated with $25 \mu \mathrm{M} \mathrm{OA}$ and $25 \mu \mathrm{M}$ HTyr (Figure 2(a)). This result can be explained, at least in part, by the reduction of HMGCR activity (Figure 4 ) and the regulatory enzyme of cholesterol synthesis, which is reasonably related to changes in the HMGCR expression. Indeed, the expression of HMGCR at both mRNA and protein levels was noticeably reduced in C6 cells upon treatment with both $\mathrm{OA}$ and HTyr.

However, the reduction of cholesterologenesis by EVOO active compounds was often less pronounced, compared with fatty acid synthesis, especially in the case of the OA and HTyr coincubation.

Incubation of C6 cells with OA or HTyr singularly or in combination caused a reduction of $\left[1-{ }^{14} \mathrm{C}\right]$ acetate incorporation into fatty acids and of their subsequent esterification into complex lipids. The strongest inhibition of labelled acetate incorporation into the phospholipid fractions, mainly into phosphatidylcholine, was observed upon $\mathrm{OA}$ and $\mathrm{HTyr}$ coincubation (Table 1).

Unlike phospholipids, TG synthesis in C6 cells seems to be poorly affected by EVOO compounds. The opposite trend has been reported to occur in rat hepatocytes, where EVOO components, almost without effect on phospholipid synthesis, greatly reduced acetate incorporation into TG $[12,13]$. These findings are in agreement with the role of hepatic cells in TG synthesis [42] and with the assumption that dietary phenolic compounds may have a protective role against hepatic steatosis $[12,13]$. Phospholipids and cholesterol are important components of biological membranes. Thus, the remarkable decrease we observed in the present study of the phospholipids and cholesterol syntheses exerted by OA and HTyr led us to suppose that EVOO components might modulate in C6 cells the shift towards membrane biogenesis instead of an accumulation of cellular neutral lipids. Actually, this hypothesis is corroborated by previous studies which indicated that fatty acids exogenously added to C6 cells may represent specific means of controlling gliomatous growth $[5,43,44]$.

Coincubation with OA and HTyr greatly reduced the incorporation of $\left[1-{ }^{14} \mathrm{C}\right]$ acetate into the individual fatty acids, in particular into palmitic acid (Figure 3), the principal final product of the de novo fatty acid synthesis. This metabolic pathway is catalyzed by two enzymatic systems working in sequence: ACC and FAS. The activity of ACC, first committing step in fatty acid biosynthesis, was reduced in C6 cells treated with OA together with HTyr. Then, the reduced ACC activity in OA-/HTyr-treated C6 cells could explain, at least in part, the decrease in the $\left[1-{ }^{14} \mathrm{C}\right]$ acetate incorporation into the whole fatty acid fraction observed in Figure 2. The molecular mechanism of this reduction was 
deepened, and our results clearly indicate that coincubation of OA and HTyr reduced either ACC mRNA abundance or protein level. Conversely, in our experimental condition, that is, $4 \mathrm{~h}$ of treatment, OA and/or HTyr incubation showed practically no effect on FAS activity and on FAS mRNA abundance and protein level. With regard to the apparent insensitivity of FAS to $4 \mathrm{~h} \mathrm{OA}$ and HTyr incubation, it is worth noting that while ACC is regulated by both shortand long-term mechanisms, only the latter is involved in FAS modulation [12, 45].

A great body of evidence indicates HTyr as a potent antioxidant, and its ability to decrease reactive oxygen species (ROS) in both in vitro and in vivo experiments has been well documented (for review, see [46]). Recent works have highlighted that ROS promote the expression of sterol regulatory element-binding proteins (SREBPs) $[47,48]$ and transcription factors involved in the upregulation of key enzymes of lipogenesis and cholesterologenesis [31, 49, 50]. In fly, treatment with an antioxidant reduces ROS, suppresses lipid droplet accumulation, and delays neurodegeneration [48]. The present study shows that, in addition to the reported antioxidant effect, HTyr supplementation to C6 cells determines an early and direct decreasing effect on fatty acid synthesis and cholesterologenesis. This effect can be at least partially ascribed to a molecular mechanism that involves the downregulation of the expression of ACC and HMGCR. This decrease is more evident when $\mathrm{OA}$ is added to the cells together with HTyr, indicating in most cases an additive effect of these EVOO components on lipid metabolism. Thus, also this aspect should be considered in determining the beneficial action of EVOO components, OA and HTyr, in brain dysfunction when alterations of lipid metabolism take place.

An important matter concerns the bioavailability of EVOO phenolic compounds. HTyr is dose-dependently absorbed in humans [51, 52]. Data on plasma phenol concentrations that can be achieved in humans after consumption of olive oil are poor and controversial [52]. This may be due to a number of factors: (i) the very variable levels of phenols found in EVOO (50-800 mg/kg) [53], (ii) the level of dietary intake of olive oil, and (iii) the method and the time chosen for plasma phenol quantification [53]. Most of HTyr is present in plasma and urine in conjugated forms, mainly glucuroconjugates, suggesting extensive first-pass intestinal/ hepatic metabolism of the ingested HTyr [54]. However, a plasma concentration of HTyr up to $15 \mu \mathrm{M}$ has been measured in humans during the first $4 \mathrm{~h}$ after ingestion of $40 \mathrm{~mL}$ of olive oil containing a considerable amount of phenols (366 mg/kg) [55]. Moreover, it has been established that HTyr is able to cross the blood-brain barrier, although it presents a low brain uptake [3]. Considering the above, the question of EVOO phenol bioavailability in the brain remains a matter of debate and the physiological relevance of these in vitro findings needs to be tested in appropriate animal models and in humans. However, even if the HTyr concentration used in the present study $(25 \mu \mathrm{M})$ could be considered a pharmacological dose, the present study indicates an early and direct downregulatory effect of HTyr on fatty acids and cholesterol syntheses.

\section{Conclusions}

Correct lipid homeostasis is essential for cell survival and performance. Thus, brain fatty acid and cholesterol synthesis are critically challenged in several neurodegenerative diseases. The modulation of the activity and expression of the key enzymes of these metabolic pathways by OA and HTyr suggest a putative role in the prevention of neurological diseases, where dysfunction of lipid metabolism is involved. In this context, the reduced ACC and HMGCR expression and activity we observed in glial cells treated with such compounds may be considered of importance.

\section{Conflicts of Interest}

The authors declare that there is no conflict of interest regarding the publication of this article.

\section{Acknowledgments}

This work was in part supported by Ministero della Salute Project GR-2013-02355646.

\section{References}

[1] E. Toledo, J. Salas-Salvadó, C. Donat-Vargas et al., "Mediterranean diet and invasive breast cancer risk among women at high cardiovascular risk in the PREDIMED trial: a randomized clinical trial," JAMA Internal Medicine, vol. 175, no. 11, pp. 1752-1760, 2015.

[2] M. Á. Martínez-González, M. Ruiz-Canela, A. Hruby, L. Liang, A. Trichopoulou, and F. B. Hu, "Intervention trials with the Mediterranean diet in cardiovascular prevention: understanding potential mechanisms through metabolomic profiling," The Journal of Nutrition, vol. 146, no. 4, pp. 913S-919S, 2016.

[3] J. Rodríguez-Morató, L. Xicota, M. Fitó, M. Farré, M. Dierssen, and R. de la Torre, "Potential role of olive oil phenolic compounds in the prevention of neurodegenerative diseases," Molecules, vol. 20, no. 3, pp. 4655-4680, 2015.

[4] L. Fernández del Río, E. Gutiérrez-Casado, A. Varela-López, and J. Villalba, "Olive oil and the hallmarks of aging," Molecules, vol. 21, no. 2, p. 163, 2016.

[5] F. Natali, L. Siculella, S. Salvati, and G. V. Gnoni, "Oleic acid is a potent inhibitor of fatty acid and cholesterol synthesis in C6 glioma cells," The Journal of Lipid Research, vol. 48, no. 9, pp. 1966-1975, 2007.

[6] P. Priore, A. Cavallo, A. Gnoni, F. Damiano, G. V. Gnoni, and L. Siculella, "Modulation of hepatic lipid metabolism by olive oil and its phenols in nonalcoholic fatty liver disease," IUBMB Life, vol. 67, no. 1, pp. 9-17, 2015.

[7] E. Lei, K. Vacy, and W. C. Boon, "Fatty acids and their therapeutic potential in neurological disorders," Neurochemistry International, vol. 95, pp. 75-84, 2016.

[8] M. Piroddi, A. Albini, R. Fabiani et al., "Nutrigenomics of extra-virgin olive oil: a review," BioFactors, vol. 43, no. 1, pp. 17-41, 2017.

[9] R. Fabiani, "Anti-cancer properties of olive oil secoiridoid phenols: a systematic review of in vivo studies," Food \& Function, vol. 7, no. 10, pp. 4145-4159, 2016. 
[10] C. C. Tangney and H. E. Rasmussen, "Polyphenols, inflammation, and cardiovascular disease," Current Atherosclerosis Reports, vol. 15, no. 5, p. 324, 2013.

[11] F. Visioli, G. Bellomo, and C. Galli, "Free radical-scavenging properties of olive oil polyphenols," Biochemical and Biophysical Research Communications, vol. 247, no. 1, pp. 60-64, 1998.

[12] P. Priore, L. Siculella, and G. V. Gnoni, "Extra virgin olive oil phenols down-regulate lipid synthesis in primary-cultured rat-hepatocytes," The Journal of Nutritional Biochemistry, vol. 25, no. 7, pp. 683-691, 2014.

[13] P. Priore, D. Caruso, L. Siculella, and G. V. Gnoni, "Rapid down-regulation of hepatic lipid metabolism by phenolic fraction from extra virgin olive oil," European Journal of Nutrition, vol. 54, no. 5, pp. 823-833, 2015.

[14] W. J. Lukiw, M. Pappolla, R. P. Pelaez, and N. G. Bazan, "Alzheimer's disease-a dysfunction in cholesterol and lipid metabolism," Cellular and Molecular Neurobiology, vol. 25, no. 3-4, pp. 475-483, 2005.

[15] A. Romano, J. B. Koczwara, C. A. Gallelli et al., "Fats for thoughts: an update on brain fatty acid metabolism," The International Journal of Biochemistry \& Cell Biology, vol. 84, pp. 40-45, 2017.

[16] C. Féart, C. Samieri, V. Rondeau et al., "Adherence to a Mediterranean diet, cognitive decline, and risk of dementia," JAMA, vol. 302, no. 6, pp. 638-648, 2009.

[17] V. Pitozzi, M. Jacomelli, M. Zaid et al., "Effects of dietary extravirgin olive oil on behaviour and brain biochemical parameters in ageing rats," British Journal of Nutrition, vol. 103, no. 11, pp. 1674-1683, 2010.

[18] D. Pantano, I. Luccarini, P. Nardiello, M. Servili, M. Stefani, and F. Casamenti, "Oleuropein aglycone and polyphenols from olive mill waste water ameliorate cognitive deficits and neuropathology," British Journal of Clinical Pharmacology, vol. 83, no. 1, pp. 54-62, 2017.

[19] S. Torres, M. Lorente, F. Rodríguez-Fornés et al., "A combined preclinical therapy of cannabinoids and temozolomide against glioma," Molecular Cancer Therapeutics, vol. 10, no. 1, pp. 90$103,2011$.

[20] I. Matias, A. S. Buosi, and F. C. A. Gomes, "Functions of flavonoids in the central nervous system: astrocytes as targets for natural compounds," Neurochemistry International, vol. 95, pp. 85-91, 2016.

[21] J. J. Volpe, K. Fujimoto, J. C. Marasa, and H. C. Agrawal, "Relation of C-6 glial cells in culture to myelin," The Biochemical Journal, vol. 152, no. 3, pp. 701-703, 1975.

[22] F. A. McMorris, "Norepinephrine induces glial-specific enzyme activity in cultured plasma glioma cells," Proceedings of the National Academy of Sciences of the United States of America, vol. 74, no. 10, pp. 4501-4504, 1977.

[23] K. A. Nave and G. Lemke, "Induction of the myelin proteolipid protein (PLP) gene in C6 glioblastoma cells: functional analysis of the PLP promotor," The Journal of Neuroscience, vol. 11, no. 10 , pp. 3060-3069, 1991.

[24] A. Quincozes-Santos, L. D. Bobermin, A. Latini et al., "Resveratrol protects C6 astrocyte cell line against hydrogen peroxide-induced oxidative stress through heme oxygenase 1 ," PLoS One, vol. 8, no. 5, article e64372, 2013.

[25] G. V. Gnoni, M. J. H. Geelen, C. Bijleveld, E. Quagliariello, and S. G. van den Bergh, "Short-term stimulation of lipogenesis by triiodothyronine in maintenance cultures of rat hepatocytes,"
Biochemical and Biophysical Research Communications, vol. 128, no. 2, pp. 525-530, 1985.

[26] E. G. Bligh and W. J. Dyer, "A rapid method of total lipid extraction and purification," Canadian Journal of Biochemistry and Physiology, vol. 37, no. 1, pp. 911-917, 1959.

[27] M. J. H. Geelen, “The use of digitonin-permeabilized mammalian cells for measuring enzyme activities in the course of studies on lipid metabolism," Analytical Biochemistry, vol. 347, no. 1, pp. 1-9, 2005.

[28] P. Priore, A. M. Giudetti, F. Natali, G. V. Gnoni, and M. J. H. Geelen, "Metabolism and short-term metabolic effects of conjugated linoleic acids in rat hepatocytes," Biochimica et Biophysica Acta (BBA) - Molecular and Cell Biology of Lipids, vol. 1771, no. 10, pp. 1299-1307, 2007.

[29] F. Damiano, R. Tocci, G. V. Gnoni, and L. Siculella, "Expression of citrate carrier gene is activated by ER stress effectors XBP1 and ATF6 $\alpha$, binding to an UPRE in its promoter," Biochimica et Biophysica Acta (BBA) - Gene Regulatory Mechanisms, vol. 1849, no. 1, pp. 23-31, 2015.

[30] L. Siculella, R. Tocci, A. Rochira, M. Testini, A. Gnoni, and F. Damiano, "Lipid accumulation stimulates the capindependent translation of SREBP-1a mRNA by promoting hnRNP A1 binding to its $5^{\prime}$-UTR in a cellular model of hepatic steatosis," Biochimica et Biophysica Acta (BBA) - Molecular and Cell Biology of Lipids, vol. 1861, no. 5, pp. 471-481, 2016.

[31] E. Scoditti, N. Calabriso, M. Massaro et al., "Mediterranean diet polyphenols reduce inflammatory angiogenesis through MMP-9 and COX-2 inhibition in human vascular endothelial cells: a potentially protective mechanism in atherosclerotic vascular disease and cancer," Archives of Biochemistry and Biophysics, vol. 527, no. 2, pp. 81-89, 2012.

[32] E. Scoditti, A. Nestola, M. Massaro et al., "Hydroxytyrosol suppresses MMP-9 and COX-2 activity and expression in activated human monocytes via PKC $\alpha$ and $\mathrm{PKC} \beta 1$ inhibition," Atherosclerosis, vol. 232, no. 1, pp. 17-24, 2014.

[33] N. Scarmeas, Y. Stern, M. X. Tang, R. Mayeux, and J. A. Luchsinger, "Mediterranean diet and risk for Alzheimer's disease," Annals of Neurology, vol. 59, no. 6, pp. 912-921, 2006.

[34] R. W. Mitchell, N. H. On, M. R. Del Bigio, D. W. Miller, and G. M. Hatch, "Fatty acid transport protein expression in human brain and potential role in fatty acid transport across human brain microvessel endothelial cells," Journal of Neurochemistry, vol. 117, no. 4, pp. 735-746, 2011.

[35] J. Zhang and Q. Liu, "Cholesterol metabolism and homeostasis in the brain," Protein \& Cell, vol. 6, no. 4, pp. 254-264, 2015.

[36] M. Madra and S. L. Sturley, "Niemann-Pick type C pathogenesis and treatment: from statins to sugars," Clinical Lipidology, vol. 5, no. 3, pp. 387-395, 2010.

[37] N. Sato and R. Morishita, "The roles of lipid and glucose metabolism in modulation of $\beta$-amyloid, tau, and neurodegeneration in the pathogenesis of Alzheimer disease," Frontiers in Aging Neuroscience, vol. 7, p. 199, 2015.

[38] R. C. Block, E. R. Dorsey, C. A. Beck, J. T. Brenna, and I. Shoulson, "Altered cholesterol and fatty acid metabolism in Huntington disease," Journal of Clinical Lipidology, vol. 4, no. 1, pp. 17-23, 2010.

[39] M. Doria, L. Maugest, T. Moreau, G. Lizard, and A. Vejux, "Contribution of cholesterol and oxysterols to the pathophysiology of Parkinson's disease," Free Radical Biology \& Medicine, vol. 101, pp. 393-400, 2016. 
[40] N. D. Perera and B. J. Turner, “AMPK signalling and defective energy metabolism in amyotrophic lateral sclerosis," Neurochemical Research, vol. 41, no. 3, pp. 544-553, 2016.

[41] P. Prasanna, A. Thibault, L. Liu, and D. Samid, "Lipid metabolism as a target for brain cancer therapy: synergistic activity of lovastatin and sodium phenylacetate against human glioma cells," The Journal of Neurochemistry, vol. 66, no. 2, pp. 710716, 1996.

[42] M. Tuohetahuntila, M. R. Molenaar, B. Spee et al., "ATGL and DGAT1 are involved in the turnover of newly synthesized triacylglycerols in hepatic stellate cells," Journal of Lipid Research, vol. 57, no. 7, pp. 1162-1174, 2016.

[43] J. E. C. Sykes and M. Lopes-Cardozo, "Effect of exogenous fatty acids on lipid synthesis, marker-enzymes, and development of glial cells maintained in serum-free culture," Glia, vol. 3, no. 6, pp. 495-501, 1990.

[44] F. Leonardi, L. Attorri, R. D. Benedetto et al., "Effect of arachidonic, eicosapentaenoic and docosahexaenoic acids on the oxidative status of C6 glioma cells," Free Radical Research, vol. 39, no. 8, pp. 865-874, 2005.

[45] C. F. Semenkovich, "Regulation of fatty acid synthase (FAS)," Progress in Lipid Research, vol. 36, no. 1, pp. 43-53, 1997.

[46] F. Echeverría, M. Ortiz, R. Valenzuela, and L. A. Videla, "Hydroxytyrosol and cytoprotection: a projection for clinical interventions," The International Journal of Biochemistry \& Cell Biology, vol. 18, no. 5, article e930, 2017.

[47] A. M. Giudetti, F. Damiano, G. V. Gnoni, and L. Siculella, "Low level of hydrogen peroxide induces lipid synthesis in BRL-3A cells through a CAP-independent SREBP-1a activation," The International Journal of Biochemistry \& Cell Biology, vol. 45, no. 7, pp. 1419-1426, 2013.

[48] L. Liu, K. Zhang, H. Sandoval et al., "Glial lipid droplets and ROS induced by mitochondrial defects promote neurodegeneration," Cell, vol. 160, no. 1-2, pp. 177-190, 2015.

[49] W. Shao and P. J. Espenshade, "Expanding roles for SREBP in metabolism," Cell Metabolism, vol. 16, no. 4, pp. 414-419, 2012.

[50] F. Damiano, A. Rochira, R. Tocci, S. Alemanno, A. Gnoni, and L. Siculella, "HnRNP A1 mediates the activation of the IRES-dependent SREBP-1a mRNA translation in response to endoplasmic reticulum stress," Biochemical Journal, vol. 449, no. 2, pp. 543-553, 2013.

[51] F. Visioli, C. Galli, F. Bornet et al., "Olive oil phenolics are dose-dependently absorbed in humans," FEBS Letters, vol. 46, no. 2-3, pp. 159-160, 2000.

[52] R. de la Torre, "Bioavailability of olive oil phenolic compounds in humans," Inflammopharmacology, vol. 16, no. 5, pp. 245247, 2008.

[53] F. Visioli and C. Galli, "Olive oil: more than just oleic acid," The American Journal of Clinical Nutrition, vol. 72, no. 3, p. $853,2000$.

[54] E. Miro-Casas, M. I. Covas, M. Farre et al., "Hydroxytyrosol disposition in humans," Clinical Chemistry, vol. 49, no. 6, pp. 945-952, 2003.

[55] M. I. Covas, K. de la Torre, M. Farré-Albaladejo et al., "Postprandial LDL phenolic content and LDL oxidation are modulated by olive oil phenolic compounds in humans," Free Radical Biology \& Medicine, vol. 40, no. 4, pp. 608-616, 2006. 


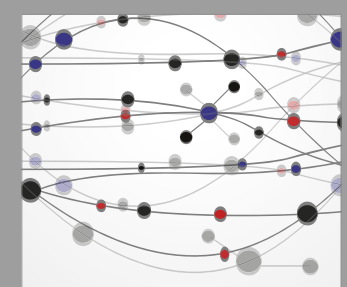

The Scientific World Journal
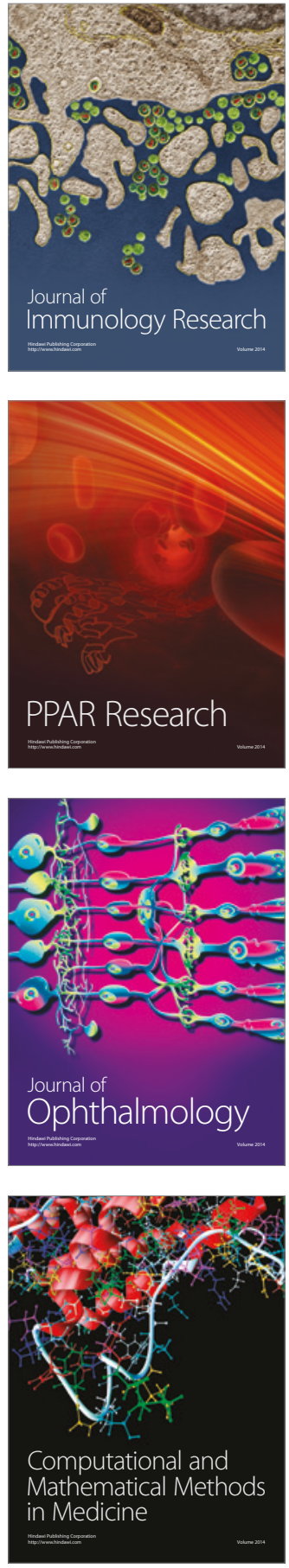

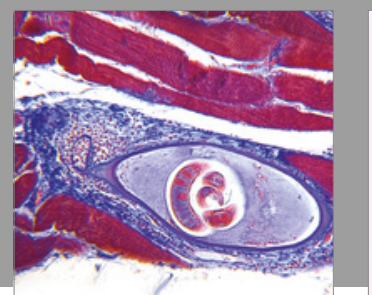

Gastroenterology Research and Practice
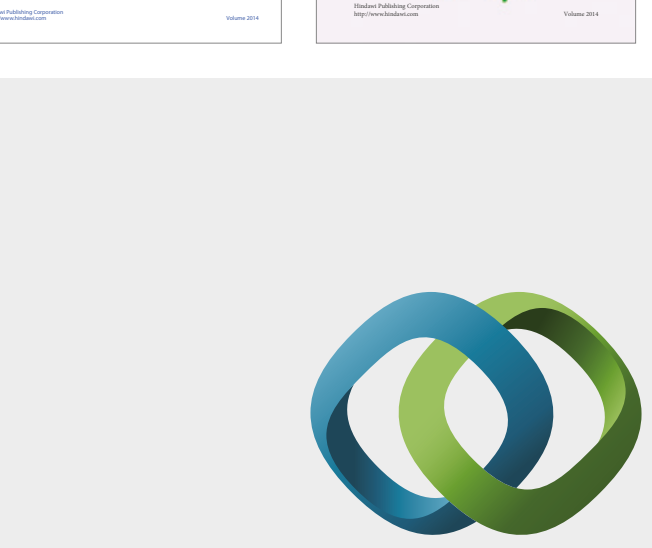

\section{Hindawi}

Submit your manuscripts at

https://www.hindawi.com
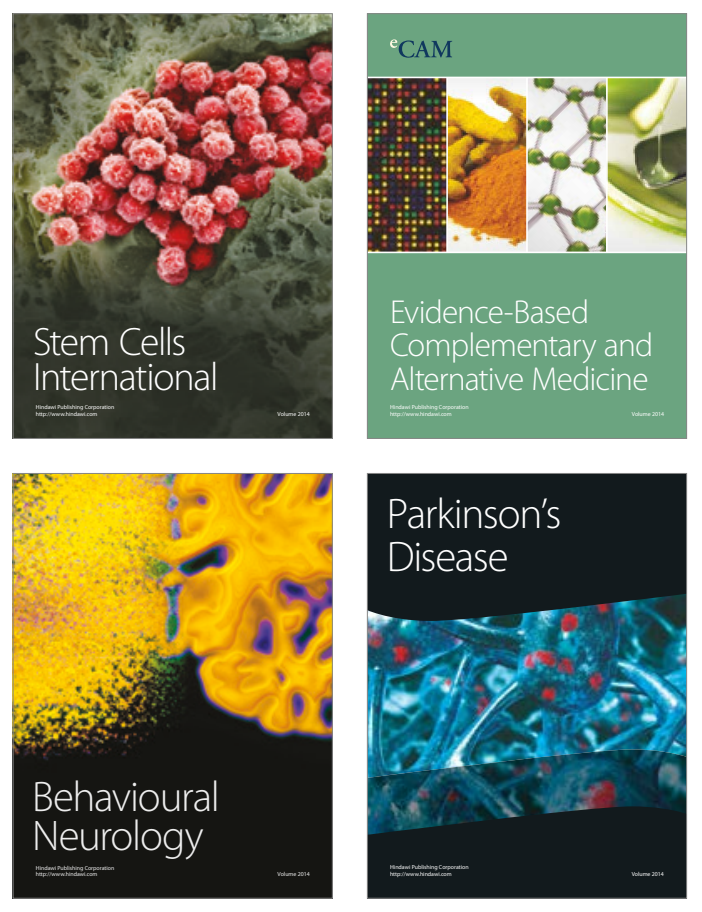
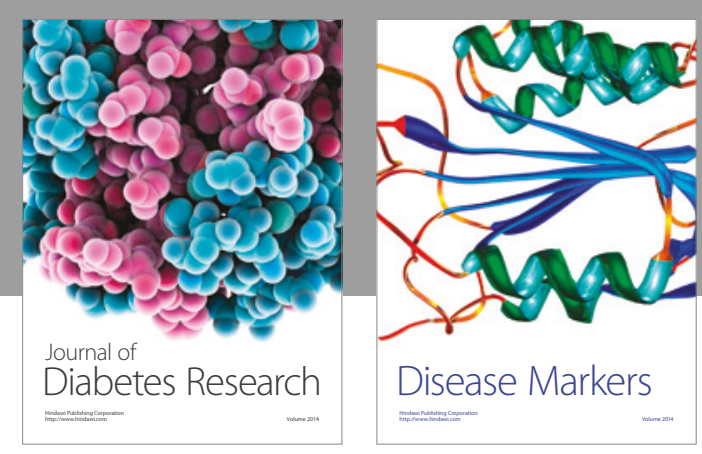

Disease Markers
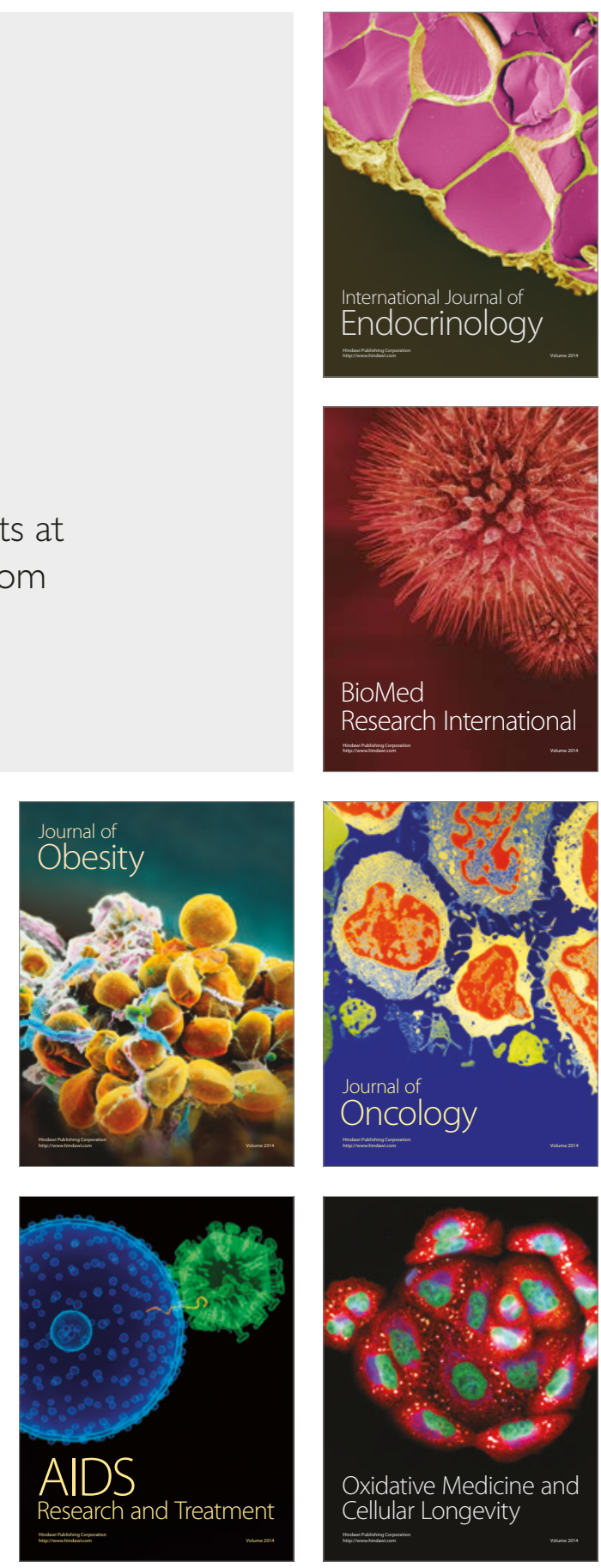